\title{
Early formation of tochilinite in CM chondrites
}

\author{
Y. ENOKIDO ${ }^{1 *}$, T. NAKAMURA ${ }^{1}$, M. MATSUMOTO ${ }^{1}$, L. $^{2}$ \\ BONAL $^{2}$ \\ 1 Tohoku University, Sendai, Miyagi 980-8578, Japan \\ (*correspondence: yuma.enokido.r8@dc.tohoku.ac.jp) \\ 2 Institut de Planétologie et d'Astrophysique de Grenoble - \\ Université Grenoble Alpes, CNRS (Grenoble - France)
}

Tochilinite is commonly observed as TochiliniteCronstedtite intergrowths (TCIs), major alteration phases, in $\mathrm{CM}$ chondrites. Formation process of tochilinite is not fully understood although several previous studies discussed its formation process (e.g., [1]). The purpose of this study is to clarify when in the aquesous alteration tochilinite fomed and the fluid condition during the tochilinite formation. We analyzed six particles of Paris CM chondrite (about $100 \mu \mathrm{m}$ in size) by synchrotron XRD, FE-SEM and TEM. The alteration degrees of these partticles were already characterized in [2]: three grains are highly altered and the others are least altered (classified as CM2.6 and 2.9, respectively, based on the alteration scale [3]).

XRD patterns of the highly altered particles are similar to those of typical CM chondrite matrices. On the other hands, XRD patterns of the least altered grains have unique features: smaller serpentine (cronstedtite) peaks and larger tochilinite peaks than those of the highly altered grains. The diffraction peak of TCIs is far smaller than that of tochilinite in the least altered grains. SEM and TEM analyses revealed that the least altered grains consist mainly of matrix mterial and fine-grained sulfur-rich aggregates. Major matrix components are amorphous silicates with embedded Fe-sulfides like GEMS (Glass Embedded Metal and Sulfide) in IDPs, indicating that these particles escaped from aqueous alteration [4]. The fine grained S-rich aggregates are composed of tochilinite of Fe end member, phyllosilicates, and some minor phases.

Our observation clarified that (1) pure Fe-tochilinite is dominant S-rich phase in the CM parent body in the initial alteration stage, (2) tochilinte formed before amorphous silicate was completely altered. Laboratory syntheses show that tochilinite forms at temperature between 120 and $160{ }^{\circ} \mathrm{C}$ [5], while amorphous silicates has easily altered to serpentine even at $25{ }^{\circ} \mathrm{C}$ in 10 days [6]. Therefore, our observation suggests that tochilinite in the $\mathrm{CM}$ chondrite may have formed at lower temperature than previously thought. [1] Pignatelli et al. (2016) MAPS 51, 785-805. [2] Bonal et al. (2019) 82th Meteoritical Society Meeting, \#2157. [3] Rubin et al. (2015) MAPS 50, 1595-1612. [4] Leroux et al. (2015) GCA 170, 247265. [5] Vacher et al. (2019) MAPS 54, 1870-1889. [6] Takigawa et al. (2019) The Astrophysical Jounal 881, 27. 